\title{
Estrutura e função: inter-relação fonoaudiológica e odontológica na reabilitação do sistema estomatognático
}

\author{
Structure and function: speech-language and dental interrelationships in the rehabilitation of the \\ stomatognathic system
}

\author{
Joab de Souza Arouche ${ }^{1^{\prime \prime}}$, Josuel Sousa Arouche ${ }^{2}$ \\ ${ }^{1}$ Universidade Federal do Amazonas, Manaus, Amazonas, Brasil. ${ }^{2}$ Centro Universitário do Norte, Manaus, Amazonas, \\ Brasil. *Autor para correspondência. E-mail: arouchejoab16@gmail.com
}

\begin{abstract}
Resumo: Introdução: O aparelho estomatognático consiste em estruturas específicas da cabeça e pescoço que desempenham funções importantes e vitais. O trabalho conjunto entre fonoaudiologia e odontologia tem sido muito evidente na literatura e mostra resultados promissores. Este estudo tem como objetivo enfatizar a contribuição da fonoaudiologia e odontologia no tratamento dos distúrbios do aparelho estomatognático, como também a importância da ação conjunta entre essas ciências. Revisão: O aparelho estomatognático, também conhecido como sistema estomatognático, recebe esse nome pela conexão entre a cavidade oral e a mandíbula. As funções deste dispositivo estão intimamente ligadas ao desenvolvimento orofacial e mandibular. A estrutura do sistema estomatognático consiste nos ossos da maxila, mandíbula e temporal. A fonoaudiologia, além de estudar cada estrutura do aparelho estomatognático, também se concentra na ordem fisiológica de cada parte estrutural, como quais são os elementos responsáveis que permitem a mastigação, que contribuem para a fonação (formação dos fonemas) e quais nervos atuam na mastigação entre outras atividades. Discussão: Para que o fonoaudiólogo e o dentista apresentem resultados clínicos eficientes, direcionados ao diagnóstico e prognóstico de pacientes com distúrbios do sistema estomatognático, é necessário um vasto conhecimento anatômico da cabeça e pescoço. Considerações finais: Com base nos relatos, foi confirmada a importância do cuidado conjunto entre fonoaudiologia e a odontologia no tratamento de patologias que comprometem o aparelho estomatognático. A fonoaudiologia é responsável pela reabilitação funcional (função) do aparelho estomatognático (mastigação, deglutição, fala, voz, fonação e respiração) e a odontologia trata da parte estrutural (cirurgia, reposicionamento da maxila e mandíbula, alinhamento e ajuste dental entre outras atividades). As principais ações conjuntas são nas especialidades ortodôntica, bucomaxilofacial, prótese e cirurgia.
\end{abstract}

Palavras-chave: aparelho estomatognático, fonoaudiologia, odontologia.

\begin{abstract}
Introduction: The stomatognathic apparatus consists of specific structures of the head and neck that perform important and vital functions. The joint work between speech therapy and dentistry has been very evident in the literature and shows promising results. This study aims to emphasize the contribution of speech therapy and dentistry in the treatment of disorders of the stomatognathic system, as well as the importance of joint action between these sciences. Review: The stomatognathic apparatus, also known as the stomatognathic system, gets its name from the connection between the oral cavity and the mandible. The functions of this device are closely linked to orofacial and mandibular development. The structure of the stomatognathic system consists of the bones of the maxilla, mandible and temporal. Speech therapy, in addition to studying each structure of the stomatognathic apparatus, also focuses on the physiological order of each structural part, such as what are the responsible elements that allow chewing, that contribute to phonation (formation of phonemes) and which nerves act in chewing among other activities. Discussion: For the speech therapist and the dentist to present efficient clinical results, directed to the diagnosis and prognosis of patients with disorders of the stomatognathic system, a vast anatomical knowledge of the head and neck is necessary. Final considerations: Based on the reports, the importance of joint care between speech therapy and dentistry in the treatment of pathologies that compromise the stomatognathic apparatus was confirmed. Speech therapy is responsible for the functional rehabilitation (function) of the stomatognathic apparatus (chewing, swallowing, speech, voice, phonation and breathing) and dentistry deals with the structural part (surgery, repositioning of the maxilla and mandible, alignment and dental adjustment among other activities). The main joint actions are in the orthodontic, buccomaxillofacial, prosthesis and surgery specialties.
\end{abstract}

Keywords: stomatognathic apparatus, speech therapy, dentistry. 


\section{Introdução}

O aparelho estomatognático consiste em estruturas específicas da cabeça e pescoço que desempenham funções importantes e vitais. O trabalho conjunto entre fonoaudiologia e odontologia tem sido muito evidente na literatura e mostra resultados promissores (Bervian \& Rodrigues, 2010). Existem inúmeras patologias que afetam o sistema estomatognático e, muitas vezes, ações conjuntas com outros profissionais são necessárias, no entanto, são enfatizadas as contribuições do fonoaudiólogo e do odontólogo neste estudo, pois são os profissionais que talvez apresentem maior conhecimento anatômico da cabeça e pescoço, com base no longo período de formação com disciplinas específicas nos cursos de graduação e pós-graduação. Uma das especialidades da fonoaudiologia é a motricidade orofacial, que estuda os músculos da cabeça, boca e pescoço. A Odontologia trata da questão estrutural do paciente com disfunção do sistema estomatognático, enquanto que a fonoaudiologia adapta, ajusta, avalia e reabilita as atividades desse sistema, promovendo uma melhor qualidade de vida para esse paciente (Aléssio, Mezzomo, \& Körbes, 2007). O papel do fonoaudiólogo nos distúrbios da cabeça e pescoço está focado no tratamento miofuncional (função). Os principais distúrbios tratados por essas duas ciências são o bruxismo, disfunção temporomandibular (DTM), fissura labiopalatina, desordens dentais, malformações congênitas, traumas de face, síndrome do respirador oral e câncer de cabeça e pescoço (Studart, 2013). Este estudo tem como objetivo enfatizar a contribuição da fonoaudiologia e odontologia no tratamento dos distúrbios do aparelho estomatognático, como também a importância da ação conjunta entre essas ciências.

\section{Revisão}

O aparelho estomatognático, também conhecido como sistema estomatognático, recebe esse nome pela conexão entre a cavidade oral e a mandíbula. As funções deste dispositivo estão intimamente ligadas ao desenvolvimento orofacial e mandibular. A estrutura do sistema estomatognático consiste nos ossos da maxila, mandíbula e temporal. O osso temporal é responsável por apoiar a mandíbula durante as funções de fala e mastigação. Na parte não móvel do aparelho estomatognático estão a maxila e os dentes superiores, ambas estruturas são inseridas no crânio. Na parte mais distal está a mandíbula que não está conectada ao osso do crânio, é o único osso móvel na cabeça e uma de suas funções é apoiar os dentes inferiores. Além da mandíbula, maxila, osso temporal, dentes, lábios e língua, o aparelho estomatognático também compreende bochechas, nervos, músculos, glândulas e artérias (Castro et al., 2012).

As funções do aparelho estomatognático estão ligadas a estímulos elétricos desencadeados pelos nervos cranianos. A articulação temporomandibular (ATM) recebe esse nome pelo fato do osso temporal se articular com a mandíbula (Douglas \& Oncins, 2011), além de auxiliar no desenvolvimento de importantes funções do aparelho estomatognático (Biasotto-Gonzalez et al., 2012).

Os dentes são essenciais para desempenhar as funções mastigatórias e de deglutição. Um indivíduo adulto possui 32 dentes divididos em 2 quadrantes (superior e inferior), com oito dentes em cada hemi-quadrante. As funções estomatognáticas são responsáveis pelo desenvolvimento e harmonia facial, as principais funções são mastigação, deglutição, respiração, fala e fonação (Cielo et al., 2016). Essas atividades são realizadas através da biomecânica funcional do aparelho estomatognático. Alterações nessas funções podem desencadear distúrbios significativos em algumas estruturas desse importante sistema. A seguir a Tabela 1 aponta importantes estruturas do aparelho estomatognático com suas inervações e principais funções

A odontologia ou medicina dentária é universal e o Brasil é um dos países em destaque nesta ciência (Rodrigues, 2015). A odontologia é a área da saúde que estuda e trata o sistema estomatognático no sentido estrutural que compreende a face, pescoço e cavidade oral, além de ossos, músculos mastigatórios, articulações (ATM), dentes, tecidos e nervos. A odontologia é dividida em diversas especialidades, como prótese, implante, estética, saúde pública, bucomaxilofacial, ortodontia, harmonia facial, odontologia legal, entre outras. Um dos deveres do dentista é realizar a cirurgia ortognática sob a competência do cirurgião bucomaxilofacial, cujo objetivo é promover a harmonia facial através do reposicionamento maxilar ou mandibular de um paciente com distúrbio orofacial. Outra importante área de atuação do dentista é a ortodontia no ajuste e reposicionamento das estruturas dentárias, para que se possa ter uma mastigação mais precisa e normal, além do foco estético. O protesista desenvolve a prótese de acordo com o modelo anatômico de cada paciente, porém o foco é estrutural e não funcional.

A odontologia ou medicina dentária é universal e o Brasil é um dos países em destaque nesta ciência $\mathrm{A}$ fonoaudiologia é a ciência que estuda a comunicação humana e todos os órgãos e estruturas relacionadas. Entre as especialidades fonoaudiológicas, destacamos a motricidade orofacial (MO) responsável pelo estudo e reabilitação muscular da face e da boca (intra / extra-oral), portanto, é competência do fonoaudiólogo habilitar, reabilitar, 
adaptar e ajustar os distúrbios miofuncionais que acometem o aparelho estomatognático. Esse profissional tem como objetivo avaliar as funções estomatognáticas e tratá-las. No entanto, para trabalhar na MO, o profissional precisa ter um bom conhecimento anatômico e fisiológico das estruturas da cabeça e pescoço. A fonoaudiologia, além de estudar cada estrutura do aparelho estomatognático, também se concentra na ordem fisiológica de cada parte estrutural, como quais são os elementos responsáveis que permitem a mastigação, que contribuem para a fonação (formação dos fonemas) e quais nervos participam da deglutição entre outras atividades.

Tabela 1. Estrutura, Inervação e Função do aparelho estomatognático.

\begin{tabular}{|c|c|c|}
\hline \multicolumn{3}{|c|}{ PRINCIPAIS MÚSCULOS DA FACE } \\
\hline ESTRUTURA & INERVAÇÃO & FUNÇÃO \\
\hline Orbicular da boca & Nervo facial (VII) & Vedamento e protrusão labial \\
\hline Levantador do lábio superior & Nervo facial (VII) & Elevação do lábio superior \\
\hline Levantador do lábio superior e asa do nariz & Nervo facial (VII) & Dilata as narinas \\
\hline Levantador do ângulo da boca & Nervo facial (VII) & Elevar o ângulo da boca \\
\hline Risório & Nervo facial (VII) & Riso \\
\hline Abaixador do ângulo da boca & Nervo facial (VII) & Retrai e puxa para baixo a comissura da boca \\
\hline Mentual & Nervo facial (VII) & Elevar a pele do mento e everter o lábio inferior \\
\hline Bucinador & Nervo facial (VII) & Puxa as comissuras labiais \\
\hline \multicolumn{3}{|c|}{ MÚSCULOS DA LÍNGUA } \\
\hline ESTRUTURA & INERVAÇÃO & FUNÇÃO \\
\hline Palatoglosso & Nervo glossofaríngeo (IX) & Elevar a língua \\
\hline Hioglosso & Nervo hipoglosso (XII) & Puxa a língua para baixo \\
\hline Genioglosso & Nervo hipoglosso (XII) & Protrusão de língua \\
\hline Estiloglosso & Nervo hipoglosso (XII) & Língua para trás \\
\hline Longitudinal superior & Nervo hipoglosso (XII) & Encurta o dorso lingual \\
\hline Longitudinal inferior & Nervo hipoglosso (XII) & Encurta o ventre lingual \\
\hline Transverso & Nervo hipoglosso (XII) & Estreitamento lingual \\
\hline Vertical & Nervo hipoglosso (XII) & achatamento lingual \\
\hline \multicolumn{3}{|c|}{ MÚSCULOS } \\
\hline ESTRUTURA & INERVAÇÃO & FUNÇÃO \\
\hline Temporal & Nervo trigêmeo $(\mathrm{V})$ & Levantamento mandibular \\
\hline Masseter & Nervo trigêmeo (V) & Levanta e projeta a mandíbula \\
\hline Pterigóideo medial & Nervo trigêmeo (V) & Levantamento mandibular \\
\hline Pterigóideo lateral & Nervo trigêmeo (V) & Abaixa a mandíbula (responsável pela abertura da boca) \\
\hline
\end{tabular}

\section{Discussão}

Para que o fonoaudiólogo e o dentista apresentem resultados clínicos eficientes, direcionados ao diagnóstico e prognóstico de pacientes com distúrbios do sistema estomatognático, é necessário um vasto conhecimento anatômico da cabeça e pescoço. Tanto a odontologia quanto os fonoaudiólogos são responsáveis pela classificação das oclusões dentárias. Essa classificação é baseada em estudos publicados pelos principais cientistas da área, como o apresentado por Angle dividido em três estágios (Chiodelli et al., 2016). O primeiro estágio foi considerado normal, usando a cúspide mesiovestibular do primeiro molar superior e o sulco mesiovestibular do primeiro molar inferior como ponto de vista (harmonia entre maxila e mandíbula), mas na segunda classificação temos a cúspide distovestibular do primeiro molar superior ocluindo no sulco mesiovesvtibular do primeiro molar inferior, essa classe apresenta possíveis alterações, como maxilar avançado ou maxilar recuado, palato estreito, contração mental, ausência de selamento labial, disfunção temporomandibular (DTM) e alterações em alguns fonemas. A classe três é conhecida por encaixar a cúspide mesiovestibular do primeiro molar superior no sulco mesiovestibular do primeiro inferior, os possíveis distúrbios orofaciais encontrados são maxila recuada ou mandíbula avançada, mordida cruzada, selamento labial impreciso, musculatura orofacial hipotônica, língua mal posicionada, mastigação com anteriorização do bolo e deglutição atípica (na primeira fase). Outra classificação foi feita por Lischer em 1910, dividida em três grupos. O primeiro grupo trata do macrognatismo e micrognatismo do tamanho da mandíbula, o segundo refere-se à neutroclusão, distoclusão e mesioclusão dos arcos dentários, enquanto o terceiro grupo trata da posição dentária referenciada por outras estruturas da boca, como dentes voltados para o lábio ou bochechas (vestibuloversão), dentes voltados para o palato (palatoversão), dentes direcionados para a língua (línguoversão), dentes na posição normal (mesioversão), dentes distantes da posição usual (distorção), dentes fora da linha média (supraversão), dentes abaixo do valor da linha média (infraversão), dentes que giram no seu próprio eixo (giroversão) e dentes que ocupam pseudolugares ou lugares que não são 
de sua origem (transposição). Assim, de acordo com cada classificação dentária, podem ocorrer inúmeras alterações orofaciais (Campos et al., 2013), por esse e outros motivos a participação do fonoaudiólogo é essencial para ajustar as funções estomatognáticas comprometidas, enfatizando o aspecto funcional. $\mathrm{O}$ dentista através da tecnologia reabilita estruturalmente os elementos do aparelho.

Quando se fala em atuar na reabilitação do aparelho estomatognático, o primeiro pensamento que surge é uma ação conjunta entre vários profissionais de saúde (Luchesi et al., 2016). As estruturas deste sistema estão ligadas a outras partes do corpo que, juntas, são responsáveis pela experiência e desenvolvimento do homem em todas as fases da vida. Algumas funções vitais são colocadas em prática pelo aparelho estomatognático, como deglutição e respiração, os elementos que compõem esse sistema também são responsáveis pela comunicação por intermédio dos órgãos fonoarticulatórios (OFAS). Os distúrbios orofaciais nem sempre são percebidos. Um exemplo é a paralisia facial parcial (em algumas partes da face); no entanto, os distúrbios da cabeça e do pescoço geralmente têm um prognóstico favorável, mas, para obter resultados efetivos, é necessário monitorar esses pacientes o mais cedo possível. As ciências que mais se destacam no tratamento de pacientes com distúrbios orofaciais e estão intimamente ligadas ao estudo do aparelho estomatognático são a fonoaudiologia e a odontologia, pois, desde a graduação, os futuros profissionais recebem todo apoio acadêmico e estágios específicos nas áreas de cabeça e pescoço.

Existem centros e clínicas dedicadas ao tratamento dos pacientes com distúrbios na região da cabeça e pescoço sob gestão conjunta entre fonoaudiólogos e odontólogos e, na maioria das vezes, o atendimento é realizado ao mesmo tempo. Os casos mais frequentes atendidos na clínica odontológica/fonoaudiológica são de pacientes diagnosticados com bruxismo, onde a odontologia visa promover o desenvolvimento de placas dentárias, evitando o atrito entre os dentes, além de ser responsável pelo diagnóstico desses pacientes, enquanto a fonoaudiologia atua nos músculos mastigatórios para reduzir a tensão muscular através de manobras. Outra ação conjunta importante entre as classes é o tratamento de pacientes com fissura labiopalatina. A participação do fonoaudiólogo é extremamente valiosa, pois quando a criança nasce com fissura, ela apresenta na maioria dos casos dificuldade em sugar e engolir o leite materno, e muitas vezes engasga (Arouche, Lopes, Santos, \& Korbes, 2020). O fonoaudiólogo adapta e ajusta a maneira como a criança suga e engole. $\mathrm{O}$ acompanhamento fonoaudiológico é realizado em todas as etapas do desenvolvimento, pois muitos apresentam dificuldades ao longo da vida na fala, voz e mastigação devido à fissura (Melgaço et al., 2002). A contribuição odontológica está presente através do desenvolvimento de próteses para o preenchimento do palato (prótese obturadora) e pela reconstrução orofacial.

O cirurgião bucomaxilofacial é o profissional responsável pela reconstrução orofacial e pela cirurgia ortognática. Para o paciente submetido à cirurgia ortognática, o fonoaudiólogo avalia estruturas e funções estomatognáticas no pré-operatório e, no pós-operatório, reabilita as funções, reposicionando a língua e estimulando a mastigação, fala, voz e deglutição (Aléssio, Mezzomo, \& Körbes, 2007). Pacientes com diastemas, apinhamento dentário, mau posicionamento dos dentes e que necessitam de tratamento ortodôntico também são monitorados pelos serviços de fonoaudiologia, nesses casos o fonoaudiólogo atua na mastigação, reposicionamento da língua e fonemas. Estudos apontam resultados promissores entre a relação FONO/ODONTO no tratamento dos distúrbios orais (Inagaki et al., 2105; Luchesi, 2016).

\section{Considerações finais}

Com base nos relatos, foi confirmada a importância do cuidado conjunto entre fonoaudiologia e a odontologia no tratamento de patologias que comprometem o aparelho estomatognático. A fonoaudiologia é responsável pela reabilitação funcional (função) do aparelho estomatognático (mastigação, deglutição, fala, voz, fonação e respiração) e a odontologia trata da parte estrutural (cirurgia, reposicionamento da maxila e mandíbula, alinhamento e ajuste dental entre outras atividades). As principais ações conjuntas são nas especialidades ortodôntica, bucomaxilofacial, prótese e cirurgia.

\section{Agradecimentos}

Os autores agradecem e dedicam esta obra a todos estudantes de odontologia e fonoaudiologia do Estado do Amazonas, em especial aos que se encontram em Manaus.

\section{Referências}

Aléssio, C. V., Mezzomo, C. L., \& Körbes, D. 2007. Intervenção fonoaudiológica nos casos de pacientes classe III com indicação à cirurgia ortognática. Arquivos em Odontologia, 43(3). 
Arouche, J. S., Lopes, D. K. C., Santos, M. A., \& Korbes, D. 2020. Ocorrência do aleitamento materno exclusivo até os seis meses de vida em indivíduos com fissura labiopalatina na rede pública de saúde do estado do amazonas: estudo preliminar. In C. R. C. Filho. Avaliação e diagnóstico da situação em saúde. Campo Grande, MS: Inovar.

Bervian, J., \& Rodrigues, R. 2010. O conhecimento dos ortodontistas sobre a atuação fonoaudiológica em respiradores bucais. $R F O, 15(3), 295-299$.

Biasotto-Gonzalez, D. A., Silva, D. S., Costa, J. M. D., Gomes, C. A. F. D. P., Hage, Y. E., Amaral, A. P., ... \& Gonzalez, T. D. O. 2012. Análise comparativa entre dois ângulos cervicais com a oclusão em crianças com e sem DTM. Revista CEFAC, 14(6), 1146-1152.

Campos, F. L. D., Vazquez, F. D. L., Cortellazzi, K. L., Guerra, L. M., Ambrosano, G. M. B., Meneghim, M. D. C., \& Pereira, A. C. 2013. A má oclusão e sua associação com variáveis socioeconômicas, hábitos e cuidados em crianças de cinco anos de idade. Revista de Odontologia da UNESP, 42(3), 160-166.

Castro, M. S. J. D., Toro, A. A. D. C., Sakano, E., \& Ribeiro, J. D. (2012). Avaliação das funções orofaciais do sistema estomatognático nos níveis de gravidade de asma. Jornal da Sociedade Brasileira de Fonoaudiologia, 24(2), 119-124.

Chiodelli, L., Pacheco, A. D. B., Missau, T. S., Silva, A. M. T. D., \& Corrêa, E. C. R. 2016. Influência da hipermobilidade articular generalizada sobre a articulação temporomandibular e a oclusão dentária: estudo transversal. CoDAS, 28(5):551-557.

Cielo, C. A., Ribeiro, V. V., Christmann, M. K., Lima, J. P. D. M., Pacheco-Rubim, A. D. B., Hoffmann, C. F., \& Mattiazzi, Â. L. (2016). Alterações de sistema estomatognático em indivíduos disfônicos. Revista CEFAC, 18(3), 613-625.

Douglas, C. R., \& Oncins, M. C. 2011. Fisiologia geral do sistema estomatognático. In H. J. Silva, D. A. Cunha (Orgs). O sistema estomatognático/anatomofisiologia e desenvolvimento. São José dos Campos, SP: Pulso.

Inagaki, L. T., de Almeida Prado, D. G., Iwamoto, A. S., Neto, J. S. P., Gavião, M. B. D., Puppin-Rontani, R. M., \& Pascon, F. M. 2015. Atuação interdisciplinar odontologia/fonoaudiologia no tratamento de paciente com cárie precoce da infância. Revista CEFAC, 17(2), 595-603.

Luchesi, K. F., de Toledo, I. P., Vieira, A. S., Meurer, B. E., Quadros, D. I., Corso, M. T., \& Texeira, L. Z. 2016. Fonoaudiologia e Odontologia na atenção básica: relato de experiência de educação em saúde. Distúrbios da Comunicação, 28(2), 388-93.

Melgaço, C. A., Di Ninno, C. Q. D. M., Penna, L. M., \& Vale, M. P. P. D. 2002. Aspectos ortodônticos/ortopédicos e fonoaudiológicos relacionados a pacientes portadores de fissuras labiopalatinas. Jornal Brasileiro de Ortodontia e Ortopedia Facial, 23-32.

Rodrigues, M. E. J. 2015. A importância da odontologia para a saúde da população. Revista Sustinere, 3(2), 191-192. Studart, L. 2013. Avaliação fonoaudiológica na cirurgia ortognática. In D. Klein, H. Justino, I Marchesan, I. Andrade, L. Brasil, M. Pinto, \& A Tessitore (Orgs.) Avaliação em motricidade orofacial: discussão de casos clínicos. São José dos Campos, SP: Pulso.

\section{Minicurrículo}

Joab Arouche. Fonoaudiólogo (UNINORTE), Especialista em Saúde Coletiva com ênfase em Cirurgia de Cabeça e Pescoço (UCAM), Mestre em Ciência e Engenharia de Materiais (UFAM), revisor de projetos submetidos a agência de fomento à pesquisa (FAPEAM), revisor de periódicos nacionais e pesquisador no laboratório de polímeros nanoestruturados (NANOPOL- UFAM).

Josuel Arouche. Técnico em prótese dentária (GUARANY), militar permanente da Força Área Brasileira (FAB) lotado no setor de odontologia do Hospital de Aeronáutica de Manaus (HAMN). Cursa o último ano de Odontologia no centro Universitário do Norte (UNINORTE), foi monitor de Saúde Coletiva odontológica.

\footnotetext{
Como citar: Arouche, J.S, \& Arouche, J.S. 2020. Estrutura e função: inter-relação fonoaudiológica e odontológica na reabilitação do sistema estomatognático. Pubsaúde, 3, a031. DOI: https:// dx.doi.org/10.31533/pubsaude3.a031

Recebido: 29 abr. 2020.

Revisado e aceito: 30 abr. 2020.

Conflito de interesse: os autores declaram, em relação aos produtos e companhias descritos nesse artigo, não ter interesses associativos, comerciais, de propriedade ou financeiros que representem conflito de interesse.

Licenciamento: Este artigo é publicado na modalidade Acesso Aberto sob a licença Creative Commons Atribuição 4.0 (CC-BY 4.0).
} 